\title{
Subject difference in exponents from circle size, numerousness, and line length
}

\section{STANLEY J. RULE, University of Alberta, Edmonton, Canada}

Magnitude estimations with no prescribed modulus were obtained for circle size, numerousness, and line length. Psychophysical power functions were fitted to individual $S$ s' responses. Exponents from the power functions were found to correlate between continua. These results are consistent with those previously found with a prescribed modulus and support the hypothesis that individual Ss exhibit a characteristic use of numbers in making magnitude estimations.

Individual exponents from psychophysical power functions obtained from magnitude estimation have been found to be related across continua. When individual exponents for different continua were subjected to an analysis of variance significant subject effects have been found for weight, taste, and smell (Jones \& Marcus, $1961)$ and for three mineral tastes in water (Bruvold \& Gaffey, 1965). The significant subject effects indicate that the average correlation between continua was greater than zero. Positive correlations of individual exponents have been found between pairs of continua for circle size, numerousness, and line length (Rule, 1966) and between circle size and visual proportion (Rule, 1968).

The magnitude estimation procedure used in all of the above studies employed a prescribed modulus. The number assigned to a standard stimulus was designated by $E$, and Ss were asked to respond to subsequent stimuli with numbers which were proportional to the reference number. Stevens (1956; Stevens \& Harris, 1962) has suggested that results from magnitude estimation are less susceptible to bias when Ss are free to determine their own modulus by assigning any number which seems appropriate to the first stimulus. Engen \& Ross (1966) have found that when no modulus was designated the number assigned to the first stimulus had little influence on the exponent, but when a modulus was prescribed the exponent was affected by the reference number.

The question arises as to whether the correlation of exponents between continua results from a bias due to employing a prescribed modulus and would disappear if the modulus were not designated, or whether the relationship is a general judgmental phenomenon related to Ss' characteristic use of numbers in magnitude estimation. The present study investigates this question by obtaining individual exponents from magnitude estimation of line length, numerousness, and circle size with no designated modulus. These continua have been investigated previously with a prescribed modulus procedure (Rule, 1966).

\section{METHOD}

Nine lines, nine circles, and nine numerousness displays were prepared on $35-\mathrm{mm}$ slides. The projected length of lines ranged from $1.69 \mathrm{in}$. to $7.84 \mathrm{in}$., and the projected diameter of circles ranged from .96 in. to $6.84 \mathrm{in}$. The numerousness displays were patterns of dots located randomly within the same circular area. The number of dots within the displays varied from 20 to 119 . The stimuli in all three sets were spaced in equal logarithmic intervals. The projected image of the stimuli appeared white on a black background. Stimuli were presented via rear projection. Exposure time was set at $1 \mathrm{sec}$ and controlled by an Alphax shutter mounted in front of a slide projector.

The Ss were 48 students enrolled in an introductory course in psychology. Participation in psychological experiments was a course requirement.

Each $\mathrm{S}$ made magnitude estimations of circle size, line length, and numerousness. No designated modulus was employed. Ss were asked to respond to the first stimulus with any number which seemed appropriate and to respond to subsequent stimuli with numbers proportional to their subjective impression of the stimulus magnitudes. A series of nine stimuli from each continuum was presented four times. With the exception of the first stimulus presented, the order of presentation was random. The stimulus for each continuum with the fifth highest physical value was presented first. All Ss received the same order of presentation. The order in which continua were presented was varied in all six possible permutations. Eight Ss received each order. The rate of presentation was one stimulus every $10 \mathrm{sec}$.

Table 1

\section{RESULTS AND DISCUSSION}

Psychophysical power functions for each of the three continua were obtained for each S. A linear least squares solution applied to the logarithmically transformed stimulus and response measures was used to fit the power functions. Each S's responses were averaged by taking geometric means. The arithmetic means and SDs of exponents for each continuum were $1.19 \pm .29$ for circle size, and $1.09 \pm .21$ for line length, and $1.00 \pm .31$ for numerousness. The average exponents are all within \pm .03 of those reported by Rule (1966) for these three continua.

Pearson product-moment correlation coefficients of exponents obtained from the first two presentations of each series of stimuli with those obtained from the last two presentations provided split-half estimates of reliability. These estimates, uncorrected for the lower number of presentations, are presented as diagonal entries in Table 1. The off-diagonal entries are correlations of Ss' exponents computed between continua. All coefficients are significantly greater than zero when tested with a test.

The correlations between continua are of the same magnitude as those previously found for the same continua with a prescribed modulus procedure. The arithmetic mean correlation is .56 for the present data and .53 for the data from the prescribed modulus procedure reported by Rule (1966).

These results indicate that the relationship between Ss' exponents from different continua is a characteristic of magnitude estimation which does not depend upon whether a modulus is prescribed. It seems reasonable that the correlations between continua reflect a component of Ss' exponents which is due to their use of the number system.

\section{REFERENCE}

BRUVOLD, W. H., \& GAFFEY, W. R. Subjective intensity of mineral tastes in water. Joumal of Experimental Psychology, 1965, 69, 369-374.

ENGEN, T., \& ROSS, B. M. Effect of reference number on magnitude estimation. Perception \& Psy chophysics, 1, 1966, 74-76.

JONES, F. N., \& MARCUS, M. J. The subject effect in judgments of subjective magnitude. Journal of Experimental Psychology, 1961,61, 40-44.

RULE, S. J. Subject differences in exponents from psychophysical power functions. Perceptual \& Motor Skills, 1966, 23, 1125-1126.

Intercorrelation of Subject Exponents

\begin{tabular}{lccc}
\hline Continuum & Circle size & Numerousness & Line length \\
\hline Circle size & $.84^{*}$ & $.61^{*}$ & $.51^{*}$ \\
Numerousness & & $.90^{*}$ & $.55^{*}$ \\
Line length & & & $.84^{*}$ \\
\hline
\end{tabular}


RULE, S. J. Subject differences in exponents for circle size and proportion. Perceptual \& Motor Skills, $1968,26,520$.

STEVENS, S. S. The direct estimation of sensory magnitudes-loudness. American Joumal of Psychology, 1956, 69, 1-25.

STEVENS, S. S., \& HARRIS, J. R. The scaling of subjective roughness and smoothness. Journal of Experimental Psychology, 1962, 64, 489-494.

\section{NOTE}

1. This research was supported by Grant No. APA 151 from the National Research Council of Canada.

\section{Are recall and recognition independent retention processes?}

\author{
ANTHONY F. GRASHA, University of \\ Cincinnati, ${ }^{2}$ Cincinnati, Ohio 45221
}

An attempt was made to equate the difficulty of recall and recognition procedures by employing a known population of stimuli (consonants), and by using, as distractors in recognition, items from previous trials. Fifty-four Ss were tested on both retention measures and on all independent variables of repetitions (one and three), retention interval $(0,6$, and $12 \mathrm{sec})$, and consonant sequence length (four and seven items). In both measures, correct response probability was directly related to number of repetitions and inversely related to retention interval duration and consonant sequence length. Under all conditions, recall was better than recognition.

Traditionally, recognition scores are generally higher than those of recall. This difference between recall and recognition retention scores has led to speculation that different storage and retrieval processes underlie the operational procedures of each task. For example, Corbelles (1967) postulated that, unlike recall, an item is stored independently of other items in recognition, and $S$ is thought to have direct access to the internal representation or memory tag of an item. A recognition response is given if the memory tag is present. A further process distinction is that $S$ is more likely to partially learn the material when a recognition test is given (McNulty, 1965). Thus a partial representation of a response (e.g., one or two letters) is presumably stored and $S$ can use this information to identify an item. In recall, partial information would not be generally sufficient for $S$ to produce a response. Finally, Deese (1963) views response selection in recognition as a process where $S$ discriminates the list membership of items that appear on the recognition sheet, whereas recall involves production as well as discrimination.

The major problem with much of the traditional work comparing these measures is that process differences have been suggested when the difficulty of the retention tasks were not equated. On a recognition test, the availability of partial response information (McNulty, 1965) and the number and availability of the response alternatives (Slamecka, 1967) are task variables which have made the recognition task easier than recall. The above studies have shown that when these factors are controlled, the traditional differences between recall and recognition scores can be reduced and eliminated. Before distinct process explanations are used to account for recall and recognition performance, it should first be established that task elements are not responsible for any observed differences. It is possible that recognition may be a process whereby $S$ covertly recalls, either completely or incompletely, a response and then matches that response to one of the alternatives on the test. Thus, recall could be considered a part of recognition and, contrary to Deese's view, both recall and recognition may involve production of a response and discrimination of whe ther it was seen before

In the present experiment, an attempt was made to equate a multiple trial recall and recognition task by employing previously learned items as distractors and by using a known and finite population of stimuli (consonants). Previously learned items were used as distractors in order to equate the type of interference in each measure. Typically such items are not used as distractors in recognition, whereas in recall, high probable intrusions on Trial $\mathrm{N}$ are items from the two previous trials (Posner, 1967). Under the assumption that the processes were not different, and that the tasks were equated, it was expected that (1) equivalent effects of the same independent variables would occur in both measures, and (2) the absolute differences between recall and recognition scores would be eliminated.

\section{SUBJECTS}

The Ss were 54 female introductory psychology students at the University of Cincinnati who participated as part of a course requirement.

\section{DESIGN}

All independent variables were manipulated within Ss. The three independent variables were: consonant sequence length, four or seven items; retention interval, 0,6, or $12 \mathrm{sec}$; and repetition condition, one and three repetitions.

Each $\mathrm{S}$ was tested in two sessions spaced 1 week apart, with one-half of the Ss having the recall measure in the first session and recognition in the second, and vice versa. On a given session, 48 experimental trials were divided into blocks of 24 trials, each using one of the consonant sequence lengths. Half of the Ss started with a seven-item block and half with a four-item block of trials. Two dummy trials, using either four- or seven-item consonant sequences, preceded the 48 experimental trials. The dummy trials were used: (a) to correct procedural mistakes before the experimental trials began, and (b) to insure that interference from two previous trials of four or seven items would be present for the first experimental trial.

The procedure for each trial follows: A slide with the word "ready" began each trial and remained on for $5 \mathrm{sec}$. This slide was followed by a consonant sequence which Ss read aloud quickly, accurately, and distinctly either once or three times. Immediately after reading the stimuli, a three-digit number was projected on the screen. During the retention interval, $S$ counted backwards by threes from this digit for either 6 or $12 \mathrm{sec}$. In the recall session, a slide with five dots was the signal to stop counting and to recall the consonants. Ss were given either $20 \mathrm{sec}$ for the four- or $35 \mathrm{sec}$ for the seven-item sequences to write each item. Guessing was allowed and they were instructed to give four or seven items on every trial. To prevent $S$ from seeing her answers from previous trials, E collected each answer sheet as it was comple ted. In the recognition session, the five dots were also the cue to stop counting. On each trial S used a list of the correct items intermixed with distractors which were the stimuli from the two previous trials. For the four- and seven-item sequences, the correct consonants were selected from a list of 12 and 21 consonants, respectively. Conditions were arranged so that $S$ could not see her answers from previous trials. The time allowed for recognition was the same as that for recall. At the end of the recall or recognition interval, a slide with the word "rest" appeared and remained on for $5 \mathrm{sec}$. The 